\title{
Utilización de pautas éticas en investigación por participantes en congreso científico estudiantil internacional, 2015
}

Usage of ethical guidelines in research by participants in international student scientific congress, 2015

\author{
Albert Estrada ${ }^{1,2}$ — https://orcid.org/0000-0002-1200-8291, Milton Méndez ${ }^{3}$ ' https://orcid.org/0000-0001-9438-732X,

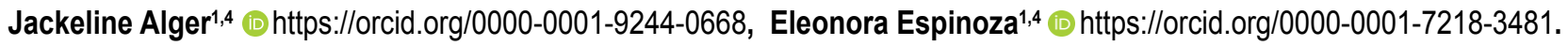

'Universidad Nacional Autónoma de Honduras (UNAH), Facultad de Ciencias Médicas (FCM), Comité de Ética en Investigación Biomédica (CEIB); Tegucigalpa, Honduras.

${ }^{2}$ Secretaría de Salud Honduras, Región Metropolitana de Salud del Distrito Central, Unidad de Atención Primaria en Salud La Cuesta; Tegucigalpa, Honduras. ${ }^{3}$ Organizacion No Gubernamental, Predicando a Cristo Sanando Vidas, Catacamas Honduras.

${ }^{4} \mathrm{UNAH}, \mathrm{FCM}$, Unidad de Investigación Científica; Tegucigalpa, Honduras.

RESUMEN. Antecedentes: El cumplimiento de normas éticas contribuye a la protección de los participantes humanos en una investigación. Objetivo: Describir la utilización de la normativa de ética en investigación por expositores de trabajos libres, XXX Congreso Científico Internacional, Federación Latinoamericana de Estudiantes de Medicina (FELSOCEM), Panamá, 2015. Metodología: Estudio descriptivo transversal. Instrumento autoadministrado previo consentimiento informado escrito. Se realizó un muestreo por conveniencia entre participantes inscritos presentando trabajo(s) libre(s). Se consideró cumplimiento de las normas éticas en investigación 1) contar con aval institucional, 2) aprobación de comité de ética en investigación (CEI) y 3) obtención de asentimiento/consentimiento informado. Se registró la información en base de datos Epilnfo vs 3.5.4 (CDC, Atlanta, EUA). El estudio fue aprobado por CEI Facultad de Ciencias Médicas UNAH y FELSOCEM. Resultados: Participaron 98 asistentes, edad promedio 22 años (desviación estándar \pm 2.53 ), 60 (61.2\%) sexo femenino, procedentes de 12 países, $73(75.2 \%)$ con más del $60 \%$ de pensum académico alcanzado. Presentaron 105 trabajos libres: $42(40.0 \%)$ casos clínicos, $63(60.0 \%)$ protocolos/trabajos de investigación (44.4\% descriptivo, $38.1 \%$ analítico, $17.5 \%$ experimental). Cumplieron tres criterios éticos de investigación 33 (31.4\%), dos criterios $39(37.1 \%)$, un criterio $26(24.8 \%)$, ningún criterio 7 (6.7\%). Discusión: El $56.2 \%$ (59) de los trabajos presentados no cumplió con las normas éticas en investigación. En época de pandemia, la revisión ética es aún más relevante. Los eventos científicos de asociaciones estudiantiles ofrecen una oportunidad de fortalecer la conducta responsable en investigación en años formativos, lo cual debe ser promovido por el comité organizador de cada congreso.

Palabras Clave: América Latina; Estudiantes de medicina; Ética en investigación; Investigación, Proyectos de investigación.

Recibido: 22-04-2021 Aceptado: 29-08-2021 Primera vez publicado en línea: 24-09-2021 Dirigir correspondencia a: Dr. Albert Estrada

Correo electrónico: albertstrada@hotmail.es

DECLARACIÓN DE RELACIONES Y ACTIVIDADES FINANCIERAS Y NO FINANCIERAS: Ninguna.

\section{INTRODUCCIÓN}

La investigación científica es el conjunto de procedimientos sistemáticos destinados a generar nuevo conocimiento en un determinado campo 0 área. ${ }^{1,2}$ En el caso de las ciencias médicas, la generación de estos conocimientos se obtiene mediante la realización de proyectos de investigación, los cuales pueden incluir la participación de seres humanos. Estos estudios deben ser metodológica y éticamente bien diseñados para evitar riesgos innecesarios en los participantes. ${ }^{3}$ La ética en investigación tiene como objetivo primordial la protección de los participantes humanos y ha evolucionado a partir de diferentes acontecimientos a través de su historia en los cuales se violentó la integridad de los participantes en investigación. Debido a estos acontecimientos se crearon diferentes normativas y códigos, entre ellos el Código de Núremberg, la Declaración de Helsinki, el Informe Belmont y las Pautas Éticas Internacionales para la Investigación Relacionada con la Salud con Seres Humanos. ${ }^{4-6}$

Los principios éticos que rigen la investigación con seres humanos se resumen en el Respeto a las personas (autonomía), la Beneficencia (no dañar) y la Justicia (en distribución de los riesgos). ${ }^{7}$ La aplicación de estos tres principios éticos básicos incorpora los siguientes procesos: 1) Garantía o aval institucional o representantes comunitarios, que expresa un compromiso institucional para cumplir con las regulaciones éticas; 2) Revisión por un Comité de Ética Institucional (CEI), el cual se ha establecido para proteger los derechos y el bienestar de los

DECLARACIÓN DE CONFLICTOS DE INTERÉS: Todos los autores son miembros o colaboradores del Comité de Ética en Investigación Biomédica (CEIB), Facultad de Ciencias Médicas UNAH (ceibunahfcm@gmail.com; ceibunahfcm@unah.edu.hn).

Forma de citar: Estrada A, Méndez M, Alger J, Espinoza E. Utilización de pautas éticas en investigación por participantes en congreso científico estudiantil internacional, 2015. Rev Méd Hondur. 2021; 89 (2): 89-95. DOI: https://doi.org/10.5377/rmh.v89i2.12366

(c) 2021 Autor(es). Artículo de acceso abierto bajo la licencia https://creativecommons.org/ licenses/by/4.0/deed.es 
participantes humanos reclutados para participar en actividades de investigación conducida bajo los auspicios de la institución a la que está afiliada; y 3) Consentimiento Informado, el cual expresa una elección voluntaria de una persona para participar en un acto médico o de investigación, basándose en una comprensión completa y profunda de sus propósitos, procedimientos, riesgos, beneficios, alternativas y cualquier otro factor que pueda afectar la decisión de participar. ${ }^{7,8}$

La Federación Latinoamericana de Estudiantes de Medicina (FELSOCEM) es una organización multinacional que involucra diferentes sociedades científicas estudiantiles. ${ }^{9} \mathrm{La}$ FELSOCEM celebra diferentes eventos científicos regionales y subregionales de manera continua y de forma rotativa entre los países latinoamericanos, como una oportunidad para sus afiliados de compartir experiencias, establecer vínculos y alianzas, entre otros. ${ }^{10,11}$ Uno de estos eventos es el denominado Congreso Científico Internacional $(\mathrm{CCl})$ el cual se celebra anualmente. En el año 2015, se celebró en Ciudad de Panamá, Panamá, en su edición número XXX bajo el tema central Retos en salud para Latinoamérica: obesidad, VIH y cáncer. Una de las actividades del congreso fue la presentación y concurso de trabajos libres en diferentes categorías, incluyendo protocolo de investigación, trabajo de investigación y caso clínico, con una amplia participación de los asistentes. Este estudio de investigación se realizó con el objetivo de describir la utilización de la normativa de ética en investigación por parte de los participantes con al menos un trabajo libre en el XXX Congreso Científico Internacional de la FELSOCEM, con el propósito de promover la implementación de estrategias para el cumplimiento de las buenas prácticas clínicas en investigación y fortalecer la investigación ética y de calidad en Latinoamérica.

\section{PARTICIPANTES Y MÉTODOS}

Se llevó a cabo un estudio descriptivo transversal sobre los participantes en el XXX Congreso Científico Internacional FELSOCEM celebrado en la Ciudad de Panamá, Panamá, los días 13 al 16 de septiembre de 2015. Se incluyeron en el estudio a los participantes debidamente inscritos en el Congreso, quienes en fases previas enviaron sus trabajos a revisión por el Comité Organizador basándose en la normativa técnicocientífica del congreso. Los participantes presentaron uno 0 más trabajos libres en las categorías Protocolo de investigación, Trabajo de investigación y Caso clínico. Solamente se invitaron a los participantes mayores de 18 años, de cualquier sexo y país de origen.

Se realizó un muestreo no probabilístico por conveniencia tratando de dar cobertura al mayor número posible de participantes. La información fue recolectada durante los tres días del evento en las instalaciones donde se realizó el Congreso (https://www.intramed.net/evento.asp?contenidoid=87627). Los participantes fueron invitados a participar en el estudio antes de presentar su trabajo libre al jurado calificador respectivo.

La información se registró por el propio participante (autoadministración), previo consentimiento informado escrito, en un documento impreso tipo cuestionario. El cuestionario incluyó tres secciones: datos sociodemográficos (edad, sexo, país de procedencia), datos de la investigación (diseño del estudio, fuente de los datos, lugar de realización de la investigación, asesoramiento, financiamiento) y ética en investigación (aval institucional, revisión por un CEl, asentimiento y/o consentimiento informado), conteniendo 18 preguntas cerradas y 5 abiertas. En los datos generales se registró si la persona que brindaba la información era autor principal o coautor, nivel del pensum académico alcanzado en la carrera de medicina, participaciones previas en el Congreso Científico Internacional FELSOCEM, tipo de universidad de procedencia, formación en cursos de buenas prácticas clínicas y existencia de un comité de ética en investigación en su universidad.

La información fue ingresada en una base de datos diseñada en Epilnfo versión 3.5.4 (CDC, Atlanta, EUA). Se obtuvieron frecuencias y porcentajes de las variables estudiadas y se realizó análisis bivariado. Para fines del análisis de los trabajos libres, protocolo y trabajo de investigación se analizaron como un solo grupo. Se consideró cumplimiento de las normas éticas en investigación la aplicación de los siguientes criterios: 1) Contar con aval institucional, 2) Contar con aprobación por un CEl, 3) Obtención de asentimiento y/o consentimiento informado en estudios con participantes humanos. Se consideró adecuada toda investigación que cumplió con estos tres requisitos.

El protocolo del estudio fue aprobado por el Comité de Ética en Investigación Biomédica (CEIB) de la Facultad de Ciencias Médicas de la Universidad Nacional Autónoma de Honduras (UNAH), Tegucigalpa. Se obtuvo autorización para la realización del estudio por parte del Comité Organizador del CCI FELSOCEM Panamá 2015. Los participantes con trabajo libre fueron invitados a participar en el estudio mediante consentimiento informado escrito. La información personal de los participantes se manejó confidencialmente.

\section{RESULTADOS}

Se invitaron a participar en el estudio a 140 asistentes del congreso, de quienes el 70.0\% (98) aceptaron participar. Entre los 42 participantes que declinaron la invitación, las razones para no participar incluyeron falta de tiempo para brindar la información $61.9 \%$ (26) y sin ninguna razón en específica 38.1\% (16)

Las características de los participantes se presentan en los Cuadros 1 y 2. De los 98 participantes, el 50.0\% (49) pertenecían al grupo de edad de 18-22 años, edad promedio 22 años (rango 18-32 años); $61.2 \%$ (60) pertenecían al sexo femenino y procedían de 12 países de América Latina, en su mayoría de Colombia $30.6 \%$ (30), Paraguay 14.3\% (14), Honduras y Bolivia, cada uno 10.2\% (10) (Cuadro 1). El 74.5\% (73) había alcanzado más del $60 \%$ de su pensum académico, $82.7 \%$ (81) ya había participado en 1-2 CCl previamente. Los 98 participantes presentaron 105 trabajos libres, 40.0\% (42) casos clínicos, 60.0\% (63) entre protocolos y trabajos de investigación (Cuadro 1).

El $57.1 \%$ (56) de los asistentes sometió sus trabajos a revisión por un CEI (Cuadro 2). La distribución de las siguientes 
Cuadro 1. Características de los participantes, XXX Congreso Científico Internacional (CCI), FELSOCEM, Panamá 2015, n=98 participantes.

\begin{tabular}{|c|c|}
\hline CARACTERÍSTICAS & $n(\%)$ \\
\hline \multicolumn{2}{|l|}{ Edad (años) } \\
\hline $18-22$ & $49(50.0)$ \\
\hline $23-27$ & $45(45.9)$ \\
\hline $28-32$ & $4(4.1)$ \\
\hline Promedio (DE) & $22( \pm 2.53)$ \\
\hline \multicolumn{2}{|l|}{ Sexo } \\
\hline Masculino & $38(38.8)$ \\
\hline Femenino & $60(61.2)$ \\
\hline \multicolumn{2}{|l|}{ Procedencia } \\
\hline Colombia & $30(30.6)$ \\
\hline Paraguay & $14(14.3)$ \\
\hline Honduras & $10(10.2)$ \\
\hline Bolivia & $10(10.2)$ \\
\hline Perú & $8(8.2)$ \\
\hline El Salvador & $8(8.2)$ \\
\hline Venezuela & $6(6.1)$ \\
\hline Panamá & $4(4.1)$ \\
\hline Ecuador & $3(3.1)$ \\
\hline México & $3(3.1)$ \\
\hline Costa Rica & $1(1.0)$ \\
\hline Guatemala & $1(1.0)$ \\
\hline \multicolumn{2}{|l|}{ Porcentaje de pensum académico } \\
\hline$<20$ & $5(5.1)$ \\
\hline $20-59$ & $20(20.5)$ \\
\hline $60-80$ & $47(47.9)$ \\
\hline$>80$ & $26(26.5)$ \\
\hline \multicolumn{2}{|c|}{$\begin{array}{l}\text { Número de participaciones previas } \\
\text { en } \mathrm{CCl}\end{array}$} \\
\hline $1-2$ & $81(82.7)$ \\
\hline $3-4$ & $5(5.1)$ \\
\hline Ninguna & $12(12.2)$ \\
\hline \multicolumn{2}{|c|}{ Tipo de trabajo libre presentado, $n=105$} \\
\hline Caso clínico & $42(40.0)$ \\
\hline Protocolo/Trabajo investigación & $63(60.0)$ \\
\hline
\end{tabular}

$\mathrm{DE}=$ desviación estándar

características entre los participantes que sometieron y los que no sometieron su trabajo a revisión a un $\mathrm{CEI}$, fue la siguiente: contar con asesor temático y metodológico $76.8 \%$ (43) versus $52.4 \%$ (22); contar con un certificado de Buenas Prácticas Clínicas, 83.9\% (47) versus $71.4 \%$ (30); contar con CEl en su universidad, $87.5 \%$ (49) versus $80.9 \%$ (34); y deseo de publicar el trabajo, $100.0 \%$ (56) versus $97.6 \%$ (41); respectivamente (Cuadro 2).

Las características de los trabajos libres se presentan en los cuadros 3 y 4 . En el cuadro 3 se presentan las características de la fuente de datos y lugar de realización del trabajo libre de
Cuadro 2. Distribución de algunas características de los participantes de acuerdo con la presentación del trabajo a un comité de ética en investigación, $X X X$ Congreso Científico Internacional (CCI), FELSOCEM, Panamá 2015, n=98 participantes.

\begin{tabular}{lcc}
\hline \multirow{2}{*}{ CARACTERÍSTICAS } & \multicolumn{2}{c}{ Presentación a un CEI } \\
& Si, $\mathbf{n = 5 6}$ & No, $\mathbf{n}=42$ \\
& $\mathbf{n}(\%)$ & $\mathbf{n}(\%)$ \\
\hline Asesoramiento recibido & $2(3.6)$ & $5(11.9)$ \\
Temático & $10(17.8)$ & $15(35.7)$ \\
Metodológico & $43(76.8)$ & $22(52.4)$ \\
Ambos & $1(1.8)$ & $0(0.0)$ \\
Ninguno & & \\
Certificado de BPC & $47(83.9)$ & $30(71.4)$ \\
Si & $9(16.1)$ & $12(28.6)$ \\
No & & \\
CEI en tu universidad & $49(87.5)$ & $34(80.9)$ \\
Si & $5(8.9)$ & $3(7.2)$ \\
No & $2(3.6)$ & $5(11.9)$ \\
No se & & \\
Desea publicar el trabajo realizado & & \\
en una revista científica & & \\
Si & $56(100.0)$ & $41(97.6)$ \\
No & $0(0.0)$ & $1(2.4)$ \\
\hline CEI = Comité de Ética en Investigación; BPC=Buena Prácticas Clínicas
\end{tabular}

acuerdo con su diseño metodológico. La historia clínica $69.1 \%$ y entrevista $47.6 \%$ fueron la fuente de datos más frecuentemente utilizada en los casos clínicos presentados. En protocolo/trabajo de investigación, también se utilizó historia clínica 9.1\%-37.5\% y entrevista $27.3 \%-39.3 \%$. En este tipo de estudio también se utilizó toma de muestras $25.0 \%-32.1 \%$ y registro de laboratorio 4.2\%-27.3\% como fuente de datos. Los estudios se realizaron en instituciones públicas más frecuentemente $63.6 \%-70.8 \%$, seguido de instituciones privadas $12.5 \%-28.6 \%$. Los estudios en la comunidad fueron poco frecuentes $2.4 \%-14.3 \%$.

Entre las razones para no presentar el trabajo libre a revisión por un CEI (43), se enunciaron las siguientes: $32.5 \%$ (14) desconocimiento, $23.2 \%$ (10) falta de tiempo, $18.6 \%$ (8) no lo considero importante, $7.0 \%$ (3) no hay un CEI en su ciudad y se realizaron estudios en suelos, $4.7 \%$ (2) falta de presupuesto, $4.7 \%$ (2) falta de asesoramiento adecuado y $2.3 \%$ (1) no tenía permiso de un docente.

El diseño metodológico de los trabajos de acuerdo con los criterios de ética de la investigación aplicados se presenta en el Cuadro 4. El criterio revisión por un CEl fue aplicado en menor frecuencia en caso clínico $45.2 \%$ (19) y en investigación tipo experimental $45.5 \%$ (5). El criterio contar con aval institucional fue aplicado en menor frecuencia en investigación tipo experimental $72.7 \%$ (8). Con respecto al criterio de obtención de asentimiento y/o consentimiento informado, excluyendo a los trabajos en los que el criterio no aplica por no incluir participantes humanos, fue aplicado con menor frecuencia en los estudios con diseño descriptivo, $68.0 \%$ (17/25) y experimental 75.0\% (6/8). Al analizar la aplicación de los tres criterios de ética en la investigación, se 
Cuadro 3. Características de la fuente de datos y lugar de realización de acuerdo con el diseño del trabajo libre, XXX Congreso Científico Internacional (CCI), FELSOCEM, Panamá 2015, n=105 trabajos libres.

\begin{tabular}{lcccc}
\hline CARACTERÍstICAS & $\begin{array}{c}\text { Caso Clínico } \\
\mathbf{n = 4 2} \\
\mathbf{n}(\%)\end{array}$ & $\begin{array}{c}\text { Descriptivo } \\
\mathbf{n}=\mathbf{2 8} \\
\mathbf{n}(\%)\end{array}$ & $\begin{array}{c}\text { Analítico } \\
\mathbf{n}=24 \\
\mathbf{n}(\%)\end{array}$ & $\begin{array}{c}\text { Experimental } \\
\mathbf{n}=11 \\
\mathbf{n}(\%)\end{array}$ \\
\hline Fuente de Datos & & & & \\
Banco de datos & $8(19.1)$ & $6(21.4)$ & $8(33.3)$ & $1(9.1)$ \\
Historia clínica & $29(69.1)$ & $7(25.0)$ & $9(37.5)$ & $1(9.1)$ \\
Entrevista & $20(47.6)$ & $11(39.3)$ & $8(33.3)$ & $3(27.3)$ \\
Toma de muestras & $10(23.8)$ & $9(32.1)$ & $6(25.0)$ & $3(27.3)$ \\
Registro laboratorio & $5(11.9)$ & $3(10.7)$ & $1(4.2)$ & $3(27.3)$. \\
Lugar & & & & \\
Institución pública & $29(69.1)$ & $19(67.8)$ & $17(70.8)$ & $7(63.6)$ \\
Institución privada & $12(28.6)$ & $4(14.3)$ & $3(12.5)$ & $2(18.2)$ \\
Escuela/Colegio & $3(7.1)$ & $3(10.7)$ & $2(8.3)$ & $2(18.2)$ \\
Comunidad & $1(2.4)$ & $4(14.3)$ & $1(4.2)$ & $1(9.1)$ \\
Laboratorio & $0(0.0)$ & $0(0.0)$ & $2(8.3)$ & $0(0.0)$ \\
\hline
\end{tabular}

*La información presentada no suma 100\% ya que algunos estudios utilizaron más de una fuente de datos y se realizaron en más de un lugar.

identificó que los tres criterios fueron aplicados en $43.8 \%$ (46) de los trabajos libres (cuadro 5). De acuerdo con el diseño metodológico, los tres criterios se aplicaron en $35.7 \%$ (15) de los casos clínicos, en $46.4 \%$ (13) de los estudios observacionales, $58.3 \%$ (14) de estudios analíticos y $36.4 \%$ (4) de estudios experimentales.

\section{DISCUSIÓN}

En este estudio hemos identificado bajo cumplimiento de las pautas éticas en investigación en una muestra de estudiantes participantes en el XXX Congreso Científico Internacional FELSOCEM celebrado en la ciudad de Panamá, Panamá, en el año 2015.

Se reportó que $43.8 \%$ (46) de los trabajos realizados por los participantes expresó contar con la revisión por parte de un CEI, carta de aval institucional y obtención de asentimiento y/o consentimiento informado de los participantes humanos en sus estudios. En el Manual del Congreso, ${ }^{12}$ se especificaban los lineamientos en metodología y ética de la investigación que debían de cumplir los trabajos libres. Sin embargo, 56.2\% (59) no cumplían uno o más criterios éticos de la investigación. Esto puede ser debido a que los estudios fueron realizados sin tomar en cuenta estos requerimientos esenciales en ética de la investigación. Existe gran diversidad en el desarrollo de la investigación para la salud y en el marco regulatorio para la protección de los participantes humanos en investigación en la región de América Latina y el Caribe. ${ }^{13,14}$ Por otra parte, la aplicación de los principios éticos de la investigación requiere de una serie de factores y procesos que coexistan y se desarrollen en un sistema en el que los investigadores (profesionales o estudiantes), patrocinadores, participantes y público en general, comprendan la ciencia y la investigación como medios para beneficiar a la humanidad. ${ }^{15}$ En nuestra región, los programas de educación en investigación y ética de la investigación son escasos o incompletos. El desarrollo de investigaciones y la oportunidad de presentarlas en eventos científicos regionales favorecen la formación del talento humano en esta temática. ${ }^{16,17}$

Cuadro 4. Características del diseño metodológico del trabajo libre y la aplicación de los criterios éticos de la investigación, XXX Congreso Científico Internacional (CCl), FELSOCEM, Panamá 2015, n=105 trabajos libres.

\begin{tabular}{|c|c|c|c|c|c|c|c|}
\hline \multirow[b]{3}{*}{ DISEÑO METODOLOGICO } & \multicolumn{7}{|c|}{ Criterios Éticos de la Investigación } \\
\hline & \multicolumn{2}{|c|}{ Revisión CEI } & \multicolumn{3}{|c|}{ Asentimiento/Consentimiento Informado } & \multicolumn{2}{|c|}{ Aval institucional } \\
\hline & $\begin{array}{c}\mathrm{Si} \\
\mathrm{n}(\%)\end{array}$ & $\begin{array}{c}\text { No } \\
n(\%)\end{array}$ & $\begin{array}{c}\mathrm{Si} \\
\mathrm{n}(\%)\end{array}$ & $\begin{array}{c}\text { No } \\
\mathrm{n}(\%)\end{array}$ & $\begin{array}{c}\text { No Aplica } \\
\text { n (\%) }\end{array}$ & $\begin{array}{c}\mathrm{Si} \\
\mathrm{n}(\%)\end{array}$ & $\begin{array}{c}\text { No } \\
n(\%)\end{array}$ \\
\hline Caso Clínico (n=42) & $19(45.2)$ & $23(54.8)$ & $36(85.7)$ & $5(11.9)$ & $1(2.4)$ & $34(81.0)$ & $8(19.0)$ \\
\hline Descriptivo ( $n=28$ ) & $19(67.9)$ & $9(32.1)$ & $17(60.7)$ & $8(28.6)$ & $3(10.7)$ & $23(82.1)$ & $5(17.9)$ \\
\hline Analítico $(n=24)$ & $19(79.2)$ & $5(20.8)$ & $20(83.3)$ & $4(16.7)$ & $0(0.0)$ & $22(91.7)$ & $2(8.3)$ \\
\hline Experimental $(n=11)$ & $5(45.5)$ & $6(54.5)$ & $6(54.5)$ & $2(18.2)$ & $3(27.3)$ & $8(72.7)$ & $3(27.3)$ \\
\hline Total & $62(59.1)$ & $43(40.9)$ & 79 (75.2) & 19 (18.1) & $7(6.7)$ & 87 (82.8) & $18(17.2)$ \\
\hline
\end{tabular}


Cuadro 5. Utilización de normas éticas internacionales de acuerdo con la aplicación de tres criterios según diseño metodológico del trabajo libre, XXX Congreso Científico Internacional FELSOCEM, Panamá 2015, n= 105 trabajos libres.

\begin{tabular}{|c|c|c|c|c|c|}
\hline \multirow[b]{2}{*}{$\begin{array}{l}\text { CRITERIOS* DE ETICA DE LA } \\
\text { INVESTIGACION }\end{array}$} & \multicolumn{5}{|c|}{ Protocolo/Trabajo de Investigación } \\
\hline & $\begin{array}{c}\text { Caso Clínico } \\
n=42 \\
n(\%)\end{array}$ & $\begin{array}{c}\text { Descriptivo } \\
n=28 \\
n(\%)\end{array}$ & $\begin{array}{c}\text { Analítico } \\
n=24 \\
n(\%)\end{array}$ & $\begin{array}{l}\text { Experimental } \\
\qquad \begin{array}{c}n=11 \\
n(\%)\end{array}\end{array}$ & $\begin{array}{c}\text { Total } \\
n=105 \\
n(\%)\end{array}$ \\
\hline Tres & $15(35.7)$ & $13(46.4)$ & $14(58.3)$ & $4(36.4)$ & $46(43.8)$ \\
\hline Dos & $20(47.6)$ & $8(28.6)$ & $9(37.5)$ & $2(18.1)$ & $39(37.1)$ \\
\hline Uno & $4(9.6)$ & $4(14.3)$ & $1(4.2)$ & $4(36.4)$ & $13(12.4)$ \\
\hline Ninguno & $3(7.1)$ & $3(10.7)$ & $0(0.0)$ & $1(9.1)$ & $7(6.7)$ \\
\hline
\end{tabular}

*Criterios: Contar con revisión por un CEl, contar con aval institucional, obtención de asentimiento y/o consentimiento informado.

En este estudio los participantes que habían recibido asesoramiento metodológico y temático sometieron para dictamen a un CEI $66.2 \%$ (43) y $33.8 \%$ (22) no lo sometieron. El acompañamiento es importante para los estudiantes de grado en la elaboración de sus trabajos de investigación. Contar con una figura de amplia experiencia en la materia que lo guie y lo apoye durante todo el proceso de elaboración del proyecto y en la redacción final del manuscrito, favorecerían las buenas prácticas en investigación. ${ }^{18}$ Se debe velar por crear lo espacios necesarios y logística en cada institución de enseñanza para la correcta interacción de docentes y estudiantes para el desarrollo de investigaciones de calidad respetando la integridad de los participantes en investigación. ${ }^{19,20}$ Aunque ambos grupos, los que sometieron y no sometieron a un $\mathrm{CEI}$, tenían un curso de Buenas Prácticas Clínicas (BPC), 83.9\% (47) y 71.4\% (30) respectivamente, la razón principal de porque no presentaban su trabajo a revisión a un $\mathrm{CEI}$ fue desconocimiento por parte de los investigadores. En un análisis de la experiencia del CEI de la Facultad de Ciencias Médicas UNAH, se hace mención sobre el desconocimiento general por parte de los investigadores de las normas éticas internacionales. ${ }^{21} \mathrm{Es}$ de destacar el hecho de que contar con un CEI en su institución no aumentó la frecuencia de someterlo al mismo. Lo que si promovió que los participantes sometieran su trabajo para dictamen a un CEI fue el deseo de publicar. Esto refleja que los participantes tenían conocimiento de que las revistas científicas indexadas incluyen dentro de sus normas de publicación los aspectos de la ética de la investigación como requisito para publicación. ${ }^{22}$

El 59.1\% (62) del total de 105 trabajos presentados en el evento se sometió a revisión por un CEl. Los estudios experimentales se presentaron con menor frecuencia a revisión $45.5 \%$ (5). Esto llama la atención debido a que en los estudios experimentales se realiza una intervención en los participantes de la investigación. Existe una necesidad de crear e implementar la aplicación de normas que regulen la investigación de acuerdo a principios éticos fundamentales, que protejan los derechos humanos, el bienestar y la seguridad de los sujetos de investigación y que aseguren, asimismo un rigor científico, especialmente en un entorno en el cual se ha denunciado la influencia creciente del mercado en la forma de hacer investigación en salud pública. ${ }^{23}$ Los CEl tienen el fin primordial de proteger los individuos que participan en investigación y para un buen desempeño deben contar con las condiciones institucionales favorables que no solo incluyan recursos logísticos y financieros, sino también incluyan la capacidad del talento humano la cual se adquiere con la experiencia y la práctica. ${ }^{24-26} \mathrm{~A}$ mayor complejidad de diseño, más exigencia para someter a dictamen ético. Algunos CEI exoneran estudios observacionales y solamente dictaminan ensayos clínicos. En nuestro estudio los estudios analíticos son los que se sometieron con mayor frecuencia a dictamen por un CEI representando el $79.2 \%$.

Con respecto a la fuente de los datos (Cuadro 3 ), la entrevista y toma de muestras clínicas fueron de las fuentes más frecuentes en los trabajos libres tipo caso clínico, $47.6 \%$ y $23.8 \%$ respectivamente y en los protocolos/trabajos de investigación $27.3 \%-39.3 \%$ y $25.0 \%-32.1 \%$, respectivamente. Este tipo de intervenciones de investigación con los participantes en un estudio, requieren de un consentimiento informado con explicación detallada de riesgos y beneficios a los que se exponen. En relación con los datos y el lugar de recolección de la información de las investigaciones, un alto porcentaje informó que se realizó en un centro de asistencia pública y de enseñanza educativa, siendo en menor número las que se practicaron en la comunidad. En el artículo de Mayta-Tristán y colaboradores, ${ }^{10}$ se hace mención de que el proceso de investigación en estudiantes de medicina de grado ha sido debilitado por la actividad asistencial de cada país. Es poco el tiempo que los estudiantes pueden dedicar a investigar acerca de los problemas prioritarios de cada comunidad. En nuestro estudio, 91.9\% (103) de los datos de las investigaciones se obtuvieron en centros asistenciales públicos o privados o en sus centros académicos.

Aunque los resultados de este estudio no pueden generalizarse a toda la región de América Latina y el Caribe, los participantes procedían de 12 países, siendo los más frecuentes Colombia $30.6 \%$ (30), Paraguay $14.3 \%$ (14) y Bolivia y Honduras $10.2 \%$ (10), cada uno. En cuanto a las características de los participantes, participaron en nuestro estudio más mujeres $61.2 \%$ (60) que hombres $38.8 \%$ (38). El promedio de edad de 22 años y la mayoría ya había cursado más del $60 \%$ del pensum de la carrera. También se destaca que la mayoría ya habían participado en un $\mathrm{CCl}$ al menos una vez $87.8 \%$ (86). Esto favorece a que este tipo de eventos sea un espacio apropiado para informar y reforzar las buenas prácticas en investigación. ${ }^{16,17}$ 
En la realización del estudio estuvimos limitados en la recolección de los datos ya que solamente un miembro del equipo participó en la recolección. En vista de que había poco tiempo entre las presentaciones, los expositores declinaban participar en el estudio por lo que la muestra se limitó a los 98 participantes que aceptaron.

En conclusión, se ha identificado que, aunque los participantes a este evento científico regional eran estudiantes que en su mayoría ya habían participado en un evento similar, tenían una capacitación en buenas prácticas clínicas y cuyos centros académicos contaban con $\mathrm{CEI}$, dos tercios de los trabajos presentados no cumplió con las normas éticas en investigación. Se recomienda que la aceptación de los trabajos libres se limite a aquellos que cumplan con las normas éticas de investigación. En el desarrollo del evento se podrían brindar capacitaciones dirigidas a aquellos aspectos que más interesaron a los participantes como ser la publicación científica. Los eventos científicos de asociaciones estudiantiles ofrecen una oportunidad de fortalecer la conducta responsable en investigación en años formativos, lo cual debe ser promovido por el comité organizador de cada evento científico. En tiempos de pandemia, la revisión ética es necesaria y aún más relevante..$^{26,27}$

\section{CONTRIBUCIONES}

Todos los autores concibieron y diseñaron el estudio. MM recolectó los datos. Todos los autores analizaron los resultados de una manera crítica, contribuyeron en la redacción del artículo, abordaron las recomendaciones editoriales y aprobaron la versión final del manuscrito.

\section{AGRADECIMIENTO}

Al Comité Organizador del Congreso Científico Internacional de la Federación Latinoamericana de Estudiantes de Medicina (FELSOCEM) Panamá 2015 por permitirnos desarrollar esta investigación con los participantes del evento.

\section{DETALLES DE AUTOR(ES)}

Albert Estrada, Médico General; albertstrada@hotmail.es Milton Méndez, Médico General; miltonmendez20@gmail.com Jackeline Alger, Médica, Doctorado en Parasitología; jackelinealger@gmail.com

Eleonora Espinoza, Médica, Maestría en Salud Pública; eleonora.espinoza@unah.edu.hn

\section{REFERENCIAS}

1. Miyahira Arakaki JM. La investigación formativa y la formación para la investigación en el pregrado. Rev Med Hered [Internet]. 2009 [citado 2 mayo 2018];20(3):119-122. Disponible en: http://www.scielo.org.pe/pdf/ $\mathrm{rmh} / \mathrm{v} 20 \mathrm{n} 3 / \mathrm{v} 20 \mathrm{n} 3 \mathrm{e} 1 . \mathrm{pdf}$

2. Monjarás Ávila AJ, Bazán Suarez AK, Pacheco- Martínez ZK, Rivera Gonzaga JA, Zamarripa Calderón JE, Cuevas Suárez CE. Diseños de Investigación. Educación y Salud Boletín Científico Instituto de Ciencias de la Salud Universidad Autónoma del Estado de Hidalgo. [Internet]. 2019[citado 12 mayo 2020];8(15):119-122. Disponible en: https://doi. org/10.29057/icsa.v8i15.4908

3. Kalichman M. Responsible conduct of research education (what, why, and does it work?). Acad Med [Internet]. 2016 [citado 4 abril 2021];91(12): e10. Disponible en: https://www.ncbi.nlm.nih.gov/pmc/articles/PMC5378670/ pdf/nihms824212.pdf

4. Asociación Médica Mundial. Declaración de Helsinki de la AMM: principios éticos para las investigaciones médicas en seres humanos. [Internet]. Helsinki: AMM; 2017. [citado 4 abril 2021]. Disponible en: https://www. wma.net/es/policies-post/declaracion-de-helsinki-de-la-amm-principioseticos-para-las-investigaciones-medicas-en-seres-humanos/

5. Comisión Nacional para la Protección de Sujetos Humanos de Investigación Biomédica y de Comportamiento (USA). La ética y la investigación en la comunidad: Informe Belmont. principios éticos y directrices para la protección de sujetos humanos de investigación. [Internet]. Washington D.C.: La Comisión; 1976. [citado 4 abril 2021]. Disponible en: https://ori. hhs.gov/education/products/mass_cphs/training_staff/RCRspan/RCRBelmontReport.htm

6. Organización Panamericana de la Salud, Consejo de Organizaciones Internacionales de las Ciencias Médicas. Pautas éticas internacionales para la investigación relacionada con la salud con seres humanos. $4^{\mathrm{a}} \mathrm{ed}$. Ginebra: CIOMS; 2016.

7. Manchola C. Tres apuestas por una bioética práctica. Rev Bioét [Internet]. 2017[citado 4 abril 2021];25(2):264-274. Disponible en: https://www. redalyc.org/pdf/3615/361552153007.pdf

8. Vega P, López R. Ética en la investigación clínica. Rev Chil Anest [Internet]. 2014[citado 4 abril 2021]; 43:361-367. Disponible en: http://revistachilenadeanestesia.cl/PII/revchilanestv43n04.19.pdf

9. Federación Latinoamericana de Sociedades Científicas de Estudiantes de Medicina. [Internet]. FELSOCEM; 2017. [citado 4 abril 2021]. Disponible en: https://www.felsocem.net/
10. Mayta-Tristán P, Peña-Oscuvilca A. Importancia de la publicación en las sociedades científicas de estudiantes de medicina del Perú: estudio preliminar. CIMEL[Internet]. 2009[citado 18 julio 2017];14(1):27-34. Disponible en: http://www.redalyc.org/articulo.oa?id=71720571005

11. Bonilla-Escobar F, Bonilla-Vélez J, López-Castillo C. Investigación médica estudiantil: Perspectiva desde Colombia. CIMEL [Internet]. 2010 [citado 18 julio 2017];15(2):12-16. Disponible en: https://www.cimel.felsocem.net/ index.php/CIMEL/article/view/131

12. Comité Organizador XXX. Congreso Científico Internacional, Panamá 2015. Bases de los concursos científicos. Boletín XXX, CCI [Internet].2015 [citado 15 junio 2021];(1):1-69. Disponible en: http://media.wix.com/ugd/4 c051c_98b216b36eec445da87f9cb46b4206c1.pdf?fbclid=IwAR3EguiZhB immlxSZZ4pXZ10H6CHT8GVQ1BnfuUAzIKHxHZnAMIliPmQW084

13. Cuervo LG, Bermúdez-Tamayo C. Desarrollo de la investigación para la salud en Latinoamérica y el Caribe. Colaboración, publicación y aplicación del conocimiento. Gac Sanit [Internet]. 2018[citado 18 abril 2021];32(3):206-208. Disponible en: http://scielo.isciii.es/scielo. php?script=sci_arttext\&pid=S0213-91112018000300206\&lng=es

14. Department of Health and Human Services, Office for Human Research Protections (USA). International Compilation of Human Research Standards [Internet]. USA: DHHS; 2020. [citado 17 abril 2021]. Disponible en: https://www.hhs.gov/ohrp/sites/default/files/2020-international-compilation-of-human-research-standards.pdf

15. Suárez Obando F. Un marco ético amplio para la investigación científica en seres humanos: más allá de los códigos y las declaraciones: la propuesta de Ezekiel J. Emanuel. Pers Bioét. [Internet]. 2015 [citado 17 abril 2021];19(2):182-197. Disponible en: http://www.scielo.org.co/scielo. php?script=sci_arttext\&pid=S0123-31222015000200002\&Ing=en. https:// doi.org/10.5294/PBI.2015.19.2.2.

16. Ortiz-Martínez $Y$, Rotela-Fisch V, Vega-Useche L. Congresos cientificos de estudiantes de medicina en Latinoamérica. Medwave [Internet]. 2016 [citado 4 abril 2021];16(11): e6804 Disponible en: https://www.medwave. cl/link.cgi/Medwave/Perspectivas/Cartas/6804.act DOI: 10.5867/medwave.2016.11.6804

17. Bados-Enríquez DM, Baquero- Marín $P$, Morales-Jiménez E. Investigación en Colombia: oportunidades que ofrecen las sociedades científicas en programas de pregrado. CIMEL. 2018;23(1):73-74. DOl: https://doi. org/10.23961/cimel.v23i1.1018

18. Espinoza E, Alger J, Padgett D, Gonzales M. Comité de Ética en Investi- 
gación Biomédica (CEIB) de la Facultad de Ciencias Médicas, Universidad Nacional Autónoma de Honduras: experiencia 2000-2010.Rev Med Hondur [Internet]. 2011[citado 2 mayo 2018];79(2).. Disponible en: http://www. bvs.hn/Honduras/UICFCM/Vol79-2-2011-CEIB.pdf

19. Caballero-Ortiz A, Arroyo-Hernández H. Mentoría en la formación de investigadores. CIMEL [Internet]. 2014 [citado 2 mayo 2018];19(1):3. Disponible en: https://www.cimel.felsocem.net/index.php/CIMEL/article/view/445/277

20. Albanes $P$, Soares $M$, Patta $F$, Marucia $B$. Tutoring and mentoring programs in Brazilian universities: A bibliometric study. Rev Psicol [Internet]. 2015 [citado 1 de marzo 201];33(1):21-56. Disponible en: http://www.scielo.org.pe/ scielo.php?script=sci_arttext\&pid=S0254-92472015000100002\&lng=es\&n $\mathrm{rm}=$ iso

21. International Committee of Medical Journal Editors. Recommendations for the Conduct, Reporting, Editing, and Publication of Scholarly Work in Medical Journals Updated December 2019. [Internet]. ICMJE; 2020. [citado 17 abril 2021]. Disponible en: http://www.icmje.org/icmje-recommendations. pdf

22. Ramírez-Hita S. Las investigaciones de salud pública en Latinoamérica: reflexiones desde el Sur global. Rev Fac Nac Salud Pública [Internet]. 2019 [citado 17 abril 2021];37(1):106-113. Disponible en: http://www.scielo.org.co/ scielo.php?script=sci_arttext\&pid=S0120-386X2019000100106\&lng=en. https://doi.org/10.17533/udea.rfnsp.v37n1a15

23. Organización Panamericana de la Salud. Estudios de casos sobre ética de la investigación internacional en salud. Washington D.C.: OPS, 2014.

24. Sierra M. La composición multidisciplinaria, pluralista y capacitada como factores clave en el funcionamiento de un comité de ética en investigación biomédica. Rev Méd Hondur. 2017; 85:130-131.

25. Alger J. Comités de ética en investigación: anotaciones sobre su competencia e independencia. Rev Méd Hondur. 2018; 86:73-74.

26. Organización Panamericana de la Salud. Orientación y estrategias para agilizar la revisión y supervisión éticas de investigaciones relacionadas con COVID-19. [Internet]. Washington D.C.: Programa Regional de Bioética Departamento de Sistemas y Servicios de Salud, OPS; 2020. [citado 22 abril 2021]. Disponible en: file:///D:/Downloads/Covid-19-orientacionestrategias-eticas-vf.pdf

27. World Health Organization. Ethics and COVID-19. [Internet]. [citado 22 abril 2021]. Ginebra: WHO; 2021. Disponible en: https://www.who.int/ teams/health-ethics-governance/diseases/covid-19
ABSTRACT. Background: Compliance with ethical standards contributes to the protection of human participants in an investigation. Objective: Describe the use of research ethics regulation by presenters of abstracts, XXX International Scientific Congress of the Latin-American Federation of the Medicine Students, Panama, 2015. Methodology: Cross-sectional descriptive study. Self-administered instrument with written informed consent. A convenience sampling was carried out among registered participants presenting abstract(s). Compliance with ethical standards in research was defined as (1) having institutional endorsement, 2) approval of an ethics research committee (ERC) and 3) obtaining informed consent/consent. The information was recorded in Epilnfo vs. 3.5.4 database (CDC, Atlanta, USA). The study was approved by ERC Faculty of Medical Sciences UNAH and FELSOCEM. Results: 98 attendees participated, average age 22 years (standard deviation \pm 2.53$), 60(61.2 \%) 12$ countries, $73(75.2 \%)$ with more than $60 \%$ of academic pensum reached. Submitted 105 abstracts: $42(40.0 \%)$ clinical cases, $63(60.0 \%)$ research protocols/abstracts (44.4\% descriptive, $38.1 \%$ analytical, $17.5 \%$ experimental). Met the three ethical research criteria $33(31.4 \%)$, two criteria $39(37.1 \%)$, one criterion $26(24.8 \%)$, no criteria $7(6.7 \%)$. Discussion: $68.6 \%$ (72) of the abstracts submitted did not meet ethical standards in research. In pandemic times, ethical review is even more relevant. Student association scientific events offer an opportunity to strengthen responsible conduct in research in formative years, which should be promoted by the organizing committee of each scientific event.

Keywords. Ethics, research; Latin America; Research; Research design; Students, medical. 